\title{
On the illposedness and stability of the relativistic heat equation
}

Cite as: J. Math. Phys. 61, 051506 (2020); https://doi.org/10.1063/1.5123393

Submitted: 05 August 2019 . Accepted: 18 April 2020 . Published Online: 12 May 2020

A. L. García-Perciante (D), and O. Reula (D)

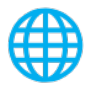

\section{Journal of}

Mathematical Physics

Young Researcher Award

Recognizing the outstanding work of early career researchers 


\title{
On the illposedness and stability of the relativistic heat equation
}

Cite as: J. Math. Phys. 61, 051506 (2020); doi: 10.1063/1.5123393

Submitted: 5 August 2019 - Accepted: 18 April 2020 •

Published Online: 12 May 2020

\begin{abstract}
A. L. García-Perciante ${ }^{1, a)}$ (D) and O. Reula ${ }^{2, b)}$ (D)

\section{AFFILIATIONS}

${ }^{1}$ Depto. de Matemáticas Aplicadas y Sistemas, Universidad Autónoma Metropolitana-Cuajimalpa, Cuajimalpa de Morelos 05348 Ciudad de México, Mexico

${ }^{2}$ Facultad de Matemática, Astronomía, Física y Computación, Universidad Nacional de Córdoba. Instituto de Física Enrique Gaviola, CONICET, Ciudad Universitaria, 5000, Córdoba, Argentina
\end{abstract}

\section{a) Author to whom correspondence should be addressed: algarcia@correo.cua.uam.mx \\ b) Electronic mail: reula@famaf.unc.edu.ar}

\begin{abstract}
In this article, we analyze, in terms of a simple example, the incompatibility of parabolic evolution and general covariance. For this, we introduce a unit time-like four-vector and study the simplest heat flux equation with respect to it. In cases where this vector field is surface forming, then the local high wave number limit shows well posedness, but as soon as that property is lost, the Cauchy problem becomes ill-posed. We also discuss how the Maxwell-Cattaneo type modification of the system renders it well posed and link the amplitude of the modification, which is related to the so-called second wave speed of the system, to the size of the failure of surface orthogonality.
\end{abstract}

Published under license by AIP Publishing. https://doi.org/10.1063/1.5123393

\section{INTRODUCTION}

In Galilean space-times, we have systems of equations describing dissipative fluids, which lead to a mixture of wave propagation and dissipative damping. They constitute hyperbolic-parabolic systems and are built, in general, following the basic example of the Navier-Stokes system. In these theories, there is a field representing the velocity of the fluid and some other quantities representing densities of energy and particle number, and then constitutive relations linking several dissipative fluxes to derivatives of some of the above fields. Together with energy, momentum, and particle number conservation, they constitute a closed evolution system.

It is well-known that the heat, or diffusion, equation which is of parabolic nature is not adequate, in general, since it allows for arbitrarily large propagation speeds. In a relativistic scenario, it is usually replaced by a Cattaneo ${ }^{1}$ type equation, a procedure that renders the system hyperbolic by introducing, without a formal derivation, a time derivative term in Fourier's law. This promotes the heat flux to a dynamical state variable and the corresponding constitutive equation to a hydrodynamic equation instead of the constraint relation status that it possesses in the conventional theory. Moreover, the Cattaneo equation can be thought of as the precursor of the widely used extended thermodynamics $^{2}$ and second order theories ${ }^{3}$ which have thoroughly been analyzed in a formal mathematical fashion. ${ }^{4,5}$ This topic has widely been addressed theoretically, experimentally, and numerically. However, the illposedness and stability of both parabolic and hyperbolic versions of the heat equation are still obscure. Moreover, the relevant role of the space-time representation in the nature of the system has practically been overlooked.

Other models have been proposed in order to attain the desired consistency between special relativity and non-equilibrium thermodynamics (see, for example, Ref. 6 and references cited therein). However, such works focus primarily on the causal nature of the heat conduction law and do not analyze the system in the more complete, covariant nature.

In this article, we analyze the dispersion relation that one is led to in the case of a Fourier heat conduction law, as well as including a heat relaxation term following Cattaneo's idea, in a covariant fashion by considering two cases. In the case where the assumed four-velocity vector is surface forming, one can establish a $3+1$ decomposition where the normal to the surfaces is precisely that four-velocity for all members of the 
foliation. In this case, the corresponding Cauchy problem is well posed, that is, solutions to the future of a given surface depend continuously on the initial data. In the generic case, where the four-velocity is not surface forming, no such foliation exists. Thus, a Cauchy problem has to be defined using an arbitrary foliation, starting from an arbitrary initial surface. The parabolic nature of the equations imply the existence of arbitrarily large propagation speeds as the wave number goes to infinity, but the surfaces under consideration cannot be aligned to these surfaces, and so perturbations influence the past of near-by points. This inconsistency is reflected in arbitrary blowups of perturbations as wave numbers increase. Thus, apart from very particular, highly symmetric, irrotational situations, the equations are ill-posed.

In order to address the point described above in a clear and brief fashion, we have organized the rest of this note as follows: In Sec. II, we state the problem by writing the heat equation as a system of partial differential equations both in a Galilean and covariant fashion and study the corresponding dispersion relations for plane waves solutions in a linear approximation first in a co-moving frame, assuming one can define such a frame (i.e., the hydrodynamic velocity forms surfaces), and second by following Hiscock et al., ${ }^{7}$ considering a boost to an arbitrary frame. The procedure is repeated in Sec. III but for a system where the Fourier law is replaced by a Cattaneo-type dynamic equation for the heat flux. The conclusions of the analysis and final remarks are included in Sec. IV.

\section{THE PARABOLIC HEAT EQUATION}

To illustrate the problem at hand, it suffices to consider the following simple Galilean system (for a discussion on Galilean space-times, see, for example, Ref. 8):

$$
\begin{aligned}
\frac{D T}{D t} & =-\frac{1}{n c_{n}} h^{a b} \nabla_{a} q_{b}, \\
q_{a} & =-\kappa \tilde{\eta}_{a}{ }^{b} \nabla_{b} T,
\end{aligned}
$$

where $\frac{D}{D t}=\left(\frac{\partial}{\partial t}+u^{a} \frac{\partial}{\partial x^{a}}\right)$ is the total (material) derivative, $c_{n}=\left(\frac{\partial e}{\partial T}\right)_{n}$ is the specific heat at constant particle number density, $\kappa$ is the thermal conductivity coefficient, and $\tilde{\eta}^{a b}$ the Euclidean flat three-metric. The covariant, relativistic, version of the system in Eq. (1) is given by

$$
\begin{aligned}
u^{a} \nabla_{a} T & =-\frac{1}{n c_{n}} h^{a b} \nabla_{a} q_{b}, \\
q_{a} & =-\kappa h_{a}^{b} \nabla_{b} T,
\end{aligned}
$$

where $u^{a}$ denotes the fluid's four velocity, and $h^{a b}=\eta^{a b}+u^{a} u^{b}$, where $\eta^{a b}$ is the flat four-metric with a $(-1,1,1,1)$ signature and a normalization, $u^{a} u_{a}=-1$. Since $u^{a}$ would, in general, be a space-time field, the equations above would have their coefficients be space-time dependent. However, it can be shown that a necessary condition for a system to be stable is that all constant coefficient systems obtained by freezing their coefficients at the values of an arbitrary space-time point must be also stable. ${ }^{9}$ Intuitively as we look for higher and higher frequency modes, we can restrict consideration to smaller and smaller patches of space-time, and so what becomes relevant in the limit are just the values of the coefficients at every limiting point. Thus, for the purposes of the analysis here performed, it suffices to pretend that the equation coefficients are constants and so amenable for Fourier transform techniques.

\section{A. Stability in the surface forming case}

If the four-vector $u^{a}$ is surface forming, that is, we can choose global surfaces whose tangent space is everywhere normal to $u^{a}$, then we can use one of them to pose a Cauchy problem, namely, to give the value of $T$ at it and obtain a unique solution to the future of such surface. If we were in such a case, we would see that perturbations propagate with arbitrary high speeds and so given any perturbation initially of compact support its solution would spread to all space-time for any arbitrarily short time to the future of our initial slice. This is in contradiction with the axiom of maximal propagation speed (the speed of light) assumed in general relativity, but actually is mathematically consistent and results in a causal propagation where causal now is in the pre general-relativity sense. Specifically, instead of maximal propagation cones, we have maximal propagation hypersurfaces. In the limit of infinite frequency, perturbations propagate along them.

Indeed, to see this look at the principal symbol of the system and analyze high frequency solutions, this suffices to assert whether the system is well posed or not. Substituting time derivatives $\left(u^{a} \nabla_{a}\right)$ by $s$ and space derivatives by $i k^{a}$, with $u^{a} k_{a}=0$, we get

$$
\begin{gathered}
s T=-\frac{i}{n c_{n}} k^{b} q_{b}, \\
q_{a}=-i \kappa k_{a} T, \quad \kappa>0,
\end{gathered}
$$

out of which we obtain the associated matrix

$$
M=\left(\begin{array}{cc}
s & \frac{i}{n c_{n}} \\
\kappa i k^{2} & 1
\end{array}\right),
$$


and from its determinant, the dispersion relation (where we have defined $\alpha=\frac{\kappa}{n c_{n}}$ )

$$
s+\alpha k^{2}=0 .
$$

Thus, the system is stable, and fluctuations decay to the future with a characteristic time $\left(\alpha k^{2}\right)^{-1}$.

\section{B. The general, not-surface forming case}

What happens when the four-velocity $u^{a}$ is not surface forming? To see this imagine in the previous case we take any space-like surface as the Cauchy surface to start our evolution. We are in trouble: the perturbations are still traveling with arbitrarily large speeds, but since our initial surface is different than that of the maximal propagation surface, signals would propagate to the future or the past of it, thus spoiling the unique solution we are seeking to construct.

To see this in formulas, consider now the construction with a different vector, that is, we choose a four-vector $n^{a}$ as being surface forming and seek to pose the Cauchy problem along these hypersurfaces. Writing

$$
g^{a b}=e^{a b}-n^{a} n^{b}, \quad n^{a} n_{a}=-1, \quad e^{a b} n_{a}=0,
$$

and

$$
u^{a}=\gamma\left(n^{a}+\beta^{a}\right), \quad \beta^{a} n_{a}=0,
$$

where $\gamma$ is the corresponding Lorentz factor for a boost with relative velocity $\beta^{a}$. In such a frame, the differential operator for plane-wave solutions is given by

$$
\tilde{\nabla}_{a}=-s n_{a}+i k_{a}, \text { with } k^{a} n_{a}=0,
$$

with the following space, time, and hydrodynamic velocity projections,

$$
n^{a} \tilde{\nabla}_{a}=s, k^{a} \tilde{\nabla}_{a}=i k^{2}, u^{a} \tilde{\nabla}_{a}=\gamma\left(s+i k_{\beta}\right),
$$

where we have defined $k_{\beta}=k_{a} \beta^{a}$. In order to obtain $h^{a b} \tilde{\nabla}_{b}$, we write

$$
h^{a b}=e^{a b}-n^{a} n^{b}+u^{a} u^{b}
$$

and thus,

$$
h^{a b} \tilde{\nabla}_{a}=i k^{b}-s n^{b}+\gamma\left(s+i k_{\beta}\right) u^{b}
$$

Finally, the system in Eq. (1) within this representation $(\nabla \rightarrow \tilde{\nabla})$ is given by

$$
\begin{gathered}
\gamma\left(s+i k_{\beta}\right) T=-\frac{1}{n c_{n}}\left(i k^{b}-s n^{b}+\gamma\left(s+i k_{\beta}\right) u^{b}\right) q_{b}, \\
q_{a}=-\kappa\left(i k_{a}-s n_{a}+\gamma\left(s+i k_{\beta}\right) u_{a}\right) T,
\end{gathered}
$$

which, considering $T$ and the scalar $\left(i k^{a}-s n^{a}\right) q_{a}$ as independent variables, results in the following associated matrix:

$$
M=\left(\begin{array}{cc}
\gamma\left(s+i k_{\beta}\right) & \frac{1}{n c_{n}} \\
-\kappa\left(s^{2}+k^{2}-\gamma^{2}\left(s+i k_{\beta}\right)^{2}\right) & 1
\end{array}\right),
$$

and dispersion relation,

$$
\left(\alpha \beta^{2} \gamma^{2}\right) s^{2}+\left(2 i k_{\beta} \gamma \alpha-1\right) \gamma s-\left(\gamma^{2} \alpha k_{\beta}^{2}+\gamma i k_{\beta}+\alpha k^{2}\right)=0 .
$$

Taking $\beta \rightarrow 0$, one gets $\gamma s+\alpha k^{2}=0$, which leads to the decay obtained in Sec. II A. For any non-zero boost, Eq. (18) has at least one root in the right half of the complex plane (using a generalization of RH criterion). In order to see this, one can normalize the equation as

$$
s^{2}+\left(p_{1}+i q_{1}\right) s+\left(p_{2}+i q_{2}\right)=0,
$$


with

$$
\begin{gathered}
p_{1}=-\left(\alpha \beta^{2} \gamma\right)^{-1}, \quad q_{1}=\frac{2 k_{\beta}}{\beta^{2}}, \\
p_{2}=-\frac{1}{\beta^{2}}\left(k_{\beta}^{2}+\frac{k^{2}}{\gamma^{2}}\right), \quad q_{2}=-\frac{k_{\beta}}{\alpha \beta^{2} \gamma} .
\end{gathered}
$$

The criterion for all roots to have negative real parts is given by $p_{1}>0$ and $q_{2}^{2}<p_{1}\left(p_{1} p_{2}+q_{1} q_{2}\right)$, which are clearly not satisfied. Thus, there is at least one root with a positive real part. Indeed, the real part of the roots is given by

$$
\operatorname{Re}\left(s_{1,2}\right)=\frac{1}{2 \beta^{2} \gamma \alpha}\{1 \pm \operatorname{Re}(\sqrt{\zeta})\}
$$

where

$$
\zeta=1+4 \alpha^{2}\left(k^{2} \beta^{2}-k_{\beta}^{2}\right)-4 \alpha i\left(\frac{k_{\beta}}{\gamma}\right)
$$

Defining, $k_{\beta}=k \beta \chi(-1 \leq \chi \leq 1)$, one can write

$$
\operatorname{Re}(\sqrt{\zeta})=\frac{1}{\sqrt{2}}\left\{1+(2 \alpha k \beta)^{2}\left(1-\chi^{2}\right)+\sqrt{\left[1+(2 \alpha k \beta)^{2}\left(1-\chi^{2}\right)\right]^{2}+(4 \alpha k \beta \gamma \chi)^{2}}\right\}^{1 / 2},
$$

which, for large values of $k$ and $\chi \neq 0$, leads to

$$
\operatorname{Re}\left(s_{1,2}\right)=\frac{1}{2 \beta^{2} \gamma \alpha}\left\{1 \pm 2 \alpha k \beta \sqrt{\left(1-\chi^{2}\right)}\right\} .
$$

Note that considering $k^{2} \beta^{2}=k_{\beta}^{2}$ (the boost is in the direction $k$ ), the expression simplifies to

$$
\operatorname{Re}\left(s_{1,2}\right)=\frac{1}{2 \alpha \beta^{2} \gamma}\left\{1 \pm \frac{1}{\sqrt{2}} \sqrt{1+\sqrt{1+\left(\frac{4 \alpha k \beta}{\gamma}\right)^{2}}}\right\},
$$

which clearly shows that one mode decays and the other one grows for any value of $k$. In this case and for large values of $k$, the growth rate of the instability increases as $\sqrt{k}$,

$$
\operatorname{Re}\left(s_{1,2}\right) \sim \pm \sqrt{\frac{k}{2 \alpha \gamma^{3} \beta^{3}}} .
$$

For general boosts, not necessarily in the direction of $\beta^{a}$, the decay is even more prominent as it grows linearly with the wave frequency.

Thus, we conclude that these systems are not only unstable, but actually ill-posed: by taking a sequence of initial data of bounded norm, but of higher and higher frequency, one can get solutions which grow arbitrarily fast no matter how small the time interval is taken. Thus, the map from initial data to solutions is not continuous. Arbitrarily close to analytic solutions, there are perfectly nice initial data points which diverge arbitrarily fast from the analytic one. Outside the very particular cases of irrotational fluids, these types of theories are thus useless.

\section{HYPERBOLIZING FLUIDS}

A simple way of rendering the system above well posed is to introduce a flow derivative in the Fourier law. In its simplest version, the modified system reads

$$
\begin{gathered}
u^{a} \nabla_{a} T=-\frac{1}{n c_{n}} h^{a b} \nabla_{a} q_{b}, \\
\varepsilon u^{b} \nabla_{b} q_{a}+q_{a}=-\kappa h_{a}^{b} \nabla_{b} T \quad \kappa>0,
\end{gathered}
$$

where Eq. (27) is the Maxwell-Cattaneo ${ }^{1}$ equation, with $\varepsilon$ being supposedly a small parameter.

As mentioned above, these ideas have been generalized and improved in order to construct causal theories for relativistic gases. ${ }^{2,4,5,10}$ Equation (27) is here considered only as a simple example in order to explore the mechanism through which the inclusion of the additional relaxation term modifies the stability and ill-posedness of the system. In Subsections III A and III B, a procedure analogous to the one shown above is carried out in order to analyze the system given by Eqs. (26) and (27). 


\section{A. Stability in the surface forming case}

Equation (27) in the fluid's frame can be written as

$$
(s \varepsilon+1) q_{a}+\kappa i k_{a} T=0,
$$

which, upon contraction with $k^{a}$, yields

$$
(s \varepsilon+1) k^{a} q_{a}+i \kappa k^{2} T=0 .
$$

In this case, the associated matrix for the longitudinal modes (the transverse components lead to trivial, stable, solutions) is given by

$$
M=\left(\begin{array}{cc}
s & \frac{i}{n c_{n}} \\
\kappa i k^{2} & 1+s \mathcal{\varepsilon},
\end{array}\right)
$$

and the corresponding dispersion relation

$$
\varepsilon s^{2}+s+\alpha k^{2}=0
$$

Since all coefficients in Eq. (31) are positive, both roots of the dispersion relation lay on the left half of the complex plane. More precisely,

$$
s_{ \pm}=\frac{1}{2 \varepsilon}\left(-1 \pm \sqrt{1-2 \alpha \varepsilon k^{2}}\right)
$$

and fluctuations decay with a characteristic time $(2 \varepsilon)^{-1}$. In the limit $\varepsilon \ll 1, s_{+}=-\frac{1}{\varepsilon}$ and $s_{-}=-\alpha k^{2}$. We have two modes, the usual one and another which decays very quickly. Indeed in the limit $\varepsilon \rightarrow 0$, we lose a root, and it goes to infinity. For small $\varepsilon$, it is arbitrarily big. The extra mode that this modification introduces is very short lived and does not affect substantially the evolution. Its initial imprint is quickly washed away, and normal diffusion follows.

\section{B. The general, not-surface forming case}

For the non-surface forming case, we shall need a minimum modification of the equations, and this should be proportional to the magnitude of the failure of the velocity vector to be surface orthogonal. Defining $\lambda=\varepsilon-\alpha \beta^{2}$, the dispersion relation in this case can be written as in Eq. (19), where now

$$
\begin{gathered}
p_{1}=(\gamma \lambda)^{-1}, \quad q_{1}=\frac{2 k_{\beta}(\varepsilon-\alpha)}{\lambda}, \\
p_{2}=\frac{\left(\gamma k_{\beta}^{2}(\alpha-\varepsilon)+\alpha k^{2}\right)}{\gamma \lambda}, \quad q_{2}=\frac{k_{\beta}}{\gamma \lambda},
\end{gathered}
$$

which reduces to Eq. (18) for $\varepsilon=0$. The criteria for stability, $p_{1}>0$ and $q_{2}^{2}<p_{1}\left(p_{1} p_{2}+q_{1} q_{2}\right)$, is then

$$
\lambda>0, \text { and } k_{\beta}^{2}<k^{2} \text {. }
$$

Note that the second condition is satisfied as long as $k \neq 0$ and $\chi \neq 1$, and thus in such cases stability is guaranteed taking $\varepsilon$ large enough such that $\varepsilon>\alpha \beta^{2}$.

The case of homogeneous perturbations $(k=0)$ leads to one pure imaginary root. The real part of the other $(\operatorname{complex})$ root is $(-\gamma \lambda)^{-1}$, which coincides with the stability criteria stated above. On the other hand, for $k \neq 0$, the real part of the roots can be written as

$$
\operatorname{Re}\left(s_{1,2}\right)=-\frac{1}{2 \lambda \gamma}[1 \pm \operatorname{Re}(\sqrt{\eta+i v})]
$$

where, for $k_{\beta}=k \beta$, one has

$$
\eta=1-4 \frac{\alpha \varepsilon}{\gamma^{2}} k^{2}, v=-\frac{4 \alpha \beta k}{\gamma}
$$

For large values of $k$, Eq. (33) leads to

$$
\operatorname{Re}\left(s_{1,2}\right) \sim-\frac{1}{2 \lambda \gamma}\left(1 \pm \beta \sqrt{\frac{\alpha}{\varepsilon}}\right) .
$$


For the stable modes $(\lambda>0)$, one obtains $\operatorname{Re}\left(s_{1,2}\right)<0$, while in the unstable case, one has $(\lambda<0)$

$$
\operatorname{Re}\left(s_{1,2}\right)=-\frac{1}{2 \lambda \gamma}\left(1 \pm \beta \sqrt{\frac{\alpha}{\varepsilon}}\right) \gtrless 0 .
$$

Note that the unstable mode is bounded and approaches a constant value for large $k$. This implies that the problem in this case is unstable but still well posed. In the general case, considering once again $k_{\beta}=k \beta \chi$, one has $v=-4 \alpha k \beta \chi / \gamma$ and

$$
\eta=1+4 \alpha k^{2}\left[\alpha \beta^{2}\left(1-\chi^{2}\right)+\varepsilon\left(\beta^{2} \chi^{2}-1\right)\right] .
$$

In this case, one obtains for large values of $k$,

$$
\operatorname{Re}\left(s_{1,2}\right) \sim-\frac{1}{2 \lambda \gamma}\left\{1 \pm \frac{\beta \chi}{\gamma} \sqrt{\frac{\alpha}{\lambda+\beta^{2} \chi^{2}\left(\frac{\alpha}{\gamma^{2}}-\lambda\right)}}\right\}<0,
$$

for $\lambda>0$, and

$$
\operatorname{Re}\left(s_{1,2}\right) \sim-\frac{1}{2 \lambda \gamma}\left\{1 \pm k \sqrt{4 \alpha\left[\beta^{2} \chi^{2}\left(\lambda-\frac{\alpha}{\gamma^{2}}\right)-\lambda\right]}\right\} \gtrless 0,
$$

for $\lambda<0$.Thus, the unstable mode grows linearly with $k$ for large values of $k$, implying that the system is ill-posed.

Note that in the stable case, the modes features a very rapid decay. Indeed, for small values of $\lambda$, from $\operatorname{Eq}$. (37), one has that $\operatorname{Re}\left(s_{1}\right)$ is proportional to $\lambda^{-1}$, while $\operatorname{Re}\left(s_{2}\right)$ is independent of $\varepsilon$. Thus, the solution decays to a sort of diffusion regime, but there is not such regime, for things cannot diffuse to arbitrary speeds. This has been studied in some cases. ${ }^{11,12}$

\section{How large does Catteno's term need to be?}

Cattaneo's term has to be larger than $\beta^{2} \alpha$, so the question is, given some vector field $u_{a}$ in some space-time, can we find a space-like foliation such that the difference between $u_{a}$ and its normal $n_{a}$ is the smallest (presumably in pointwise norm) and how big is this difference?

In principle, since $\beta<1$, we could take just this upper limit, which would give the speed of light as propagation speed of this new mode. In many cases this might not be necessary, and we could choose smaller speeds.

The measure of the local failure of surface forming for a given one-form field is given by the twist form,

$$
w_{a b c}:=u_{[a} \nabla_{b} u_{c} .
$$

Note that this is independent of any metric or torsion free connection. If $w_{a b c}$ is different from zero, it means that there is no pair of functions $(f, \tau)$ such that $f u_{a}=\nabla_{a} \tau$. In principle, one should be able to estimate the norm of $\beta_{a}=\frac{1}{\gamma} u_{a}-n_{a}$ in terms of some norm in $w_{a b c}$. This is a very difficult task since the problem is of a global nature. Consider, for instance, the case where we take $u_{a}=\gamma\left(1,0, \beta_{0}, 0\right)$ in Minkowski space-time in polar coordinates, $(t, r, \phi, z)$ with $\beta_{0}$ being a constant smaller than unity. Here, $\gamma=\left(1-\beta_{0}^{2} / r^{2}\right)^{-\frac{1}{2}}$. This form is clearly non-surface-forming: if we follow the normal planes along $\phi$, we do not arrive at the same place after a turn around the $z$ axis. Nevertheless, locally, it is possible and the only place were $w_{a b c}$ in non-vanishing is at the origin. The bound on $\beta$ in terms of $w$ cannot be local. If one relaxes $\beta_{0}$ to be a function of $r$ and writes for simplicity $u_{a}=\gamma\left(1,0, \beta_{0}(r) r, 0\right)$, then one can have it vanish near the origin (and so $w$ ). In this case, the bound is given by

$$
\left|\beta_{0}\right| \leq \frac{1-e^{-r \max |w|}}{1+e^{-r \max |w|}} .
$$

This follows from the fact that the only non-vanishing component of $w$ is $w_{t r \phi}=\gamma^{2} \partial_{r}\left(\beta_{0} r\right)$.

As another illustrative example, one can allow a modulation on $\phi$ such that one can turn around the circle and correctly paste the planes. In this case, the lack of surface forming comes from a lack of suitability in the $r$ component. Indeed, taking for simplicity the limit of small speeds, with $u_{a}=\left(1,0, \beta_{0}(r) r \cos (\phi), 0\right)$, it can be seen that surfaces which glue well in the $\phi$ direction are possible, namely, those given by $\tau=t+\beta_{0}(r) r \sin (\phi)$. However, they generate an $r$ component, $\partial_{r} \tau=\partial_{r}\left(\beta_{0} r\right) \sin (\phi)$, which leads to the same bound as above (in the limit of small velocities). Thus, one can see that is an intriguing problem where local partial differential equations theory has to be analyzed in a global context.

\section{FINAL REMARKS}

In this article, we have specifically addressed the question of whether a covariant heat equation, derived from either a Fourier or Cattaneo type relation with the help of an external four-vector field, leads to a physically sound theory. In particular, both schemes were thoroughly inspected for stability and well-posedness, using a method analogous to the one applied in Ref. 7, in two relevant scenarios: when the fluid's 
TABLE I. Summary of the results on stability and well-posedness of the relativistic heat equation.

\begin{tabular}{lll}
\hline \hline & Comoving frame & \multicolumn{1}{c}{ Boosted frame } \\
\hline Fourier & Stable well-posed & $\begin{array}{l}\text { Unstable, ill-posed } \\
\text { Stable for large } \varepsilon \text { unstable for small } \varepsilon\end{array}$ \\
Cattaneo & Stable well-posed & $\begin{array}{l}\text { well - posed if }|\chi|=1 \\
\text { ill-posed if }|\chi| \neq 1\end{array}$ \\
\hline \hline
\end{tabular}

velocity forms surfaces and when phenomena such as rotation do not allow for a particle frame to be utilized as a base for space-time $3+1$ decomposition. The results are summarized in Table I.

Note that stability in the general case depends on the magnitude of the unspecified parameter $\varepsilon$. Moreover, one could argue that under a close to equilibrium assumption, such a parameter shall remain small when compared to the parameters of the system. However, the behavior of the perturbations depend strongly on the ratio $\frac{\varepsilon}{\alpha \beta^{2}}$ and, in particular, are only well behaved when such a quantity exceeds unity. In this sense, it is worthwhile to note that the kinetic theory of gases presents, at least within the standard Chapman-Enskog program, some inconsistencies in the derivation of such term ${ }^{13}$ and thus, even though phenomenologically, one can fix the value of $\varepsilon$ arbitrarily, the soundness of such theory intuitively relies on considering only small values for it. Moreover, one can show that in the Knudsen parameter expansion, the relaxation term included in the Cattaneo equation is of higher order in such a parameter and, thus, belongs in the Burnett regime equations. On the other hand, implementing a moment expansion, one can justify the structure of Eq. (27) from microscopic grounds; however, as argued in Ref. 14, the resulting transport equations feature terms of different orders and require the introduction of an ordering scheme, in which the inconsistency exhibited in Ref. 13 will once again emerge.

\section{ACKNOWLEDGMENTS}

O.R. acknowledges financial support from Conicet, SeCyT-UNC, and MinCyT-Argentina.

\section{REFERENCES}

${ }^{1}$ C. Cattaneo, "Sur une forme de I'équation de la chaleur éliminant le paradoxe d’une propagation instantanée," C. R. Acad. Sci. 247, 431-433 (1958).

${ }^{2}$ D. Jou, J. Casas-Vázquez, and G. Lebon, Extended Irreversible Thermodynamics (Springer, 1996).

${ }^{3}$ W. Israel and J. M. Stewart, “Transient relativistic thermodynamics and kinetic theory," Ann. Phys. 118, 341-372 (1979).

${ }^{4}$ R. Geroch, "Relativistic theories of dissipative fluids," J. Math. Phys. 36, 4226-4241 (1995).

${ }^{5}$ R. Geroch and L. Lindblom, "Causal theories of dissipative relativistic fluids," Ann. Phys. 207, 394-416 (1991).

${ }^{6}$ S.-N. Li and B.-Y. Cao, "Lorentz covariance of heat conduction laws and a lorentz-covariant heat conduction model,” Appl. Math. Modell. 40, 5532-5541 (2016).

${ }^{7}$ W. A. Hiscock and L. Lindblom, "Generic instabilities in first-order dissipative relativistic fluid theories," Phys. Rev. D 31, 725 (1985).

${ }^{8}$ H. Weyl, Space Time Matter (Dover Publications, 1952).

${ }^{9}$ G. Strang, "Necessary and sufficient condition for well-posed cauchy problems," J. Differ. Equations 2, 107-114 (1966).

${ }^{10}$ R. Geroch and L. Lindblom, "Dissipative relativistic fluid theories of divergence type," Phys. Rev. D 41, 1855 (1990).

${ }^{11}$ G. Nagy, O. Ortiz, and O. Reula, “The behavior of hyperbolic heat equations' near their parabolic limits," J. Math. Phys. 35, 4334 (1994).

${ }^{12}$ H. O. Kreiss, G. Nagy, O. Ortiz, and O. Reula, “Global existence and exponential decay for hyperbolic dissipative relativistic fluid theories,” J. Math. Phys. 38, 5275-5279 (1997).

${ }^{13}$ A. L. Garcia-Perciante, H. Mondragon-Suarez, D. Brun-Battistini, and A. Sandoval-Villalbazo, "On the stability problem in special relativistic thermodynamics: Implications of the chapman-enskog formalism," J. Stat. Phys. 160, 760 (2015).

${ }^{14}$ L. S. Garcia-Colin, R. M. Velasco, and F. J. Uribe, “Inconsistency in the moment's method for solving the Boltzmann equation,” J. Non-Equilib. Thermodyn. 29, 257-277 (2005). 